\title{
Magnetic Properties and Electronic Structure of $\mathrm{ThCo}_{4} \mathrm{~B}$
}

\author{
Mohammed Said Mohammed Abu-Elmagd ${ }^{1}$, Samy Hashim Aly ${ }^{2}$, Sherif Yehia ${ }^{3}$ \\ ${ }^{1}$ Physics, Higher Institute of Engineering, Shourok Academy, Cairo, Egypt \\ ${ }^{2}$ Physics, Faculty of Science at Damietta, Mansoura University, New Damietta, Egypt \\ ${ }^{3}$ Physics, Faculty of Science, Helwan University, Cairo, Egypt \\ Email: abu_elmagd20004@yahoo.com
}

Received March 17, 2012; revised April 20, 2012; accepted April 29, 2012

\begin{abstract}
We present density functional theory DFT $a b$ initio calculation of the electronic and magnetic properties of $\mathrm{ThCo}_{4} \mathrm{~B}$ compound using the self-consistent full-potential linearized augmented plane wave (FPLAPW) method as implemented in the Wien2k package. The influence of the local environment on the Co magnetic moments is discussed by comparing the magnetic and electronic properties of $\mathrm{ThCo}_{4} \mathrm{~B}$ to its parent $\mathrm{ThCo}_{5}$ compound. The total magnetic moment in these two compounds is dominated by the Co moment. The Spin orbit interaction affects the electronic structure and spin-density maps of the p-state of Th.
\end{abstract}

Keywords: $\mathrm{ThCo}_{4} \mathrm{~B}$; Electronic Structure; Spin Density Map

\section{Introduction}

Several studies have been reported on substituted R$\mathrm{Co}_{5-x} \mathrm{X}_{x}$ compounds $(\mathrm{R}=$ lanthanide, $\mathrm{Y}$ or $\mathrm{Th})$ in order to investigate the effect of metalloids such as $\mathrm{X}=\mathrm{Al}, \mathrm{Ga}, \mathrm{Si}$, $\mathrm{B}$ on the physical properties of these systems [1-3]. In particular, there have been several studies on the structural and magnetic properties of the $\mathrm{RCo}_{4} \mathrm{~B}$ compounds [4-11]. The interest in these compounds was mainly because of their moderate hard magnetic properties with less content of the expensive Co than in $\mathrm{RCo}_{5}$ systems.

Boron-for-cobalt substitution gives rise to new structures with general formula $\mathrm{R}_{n+1} \mathrm{Co}_{3 n+5} \mathrm{~B}_{2 n}$ derived from the $\mathrm{CaCu}_{5}$ structure type. The $\mathrm{RCo}_{4} \mathrm{~B}$ compounds with non-magnetic $\mathrm{R}$ elements exhibit unique magnetic properties governed by the Co sublattice only. Their magnetic behavior differs from that of the other $\mathrm{RCo}_{4} \mathrm{~B}$ standards, and they exhibit large magnetocrystalline anisotropy. The $\mathrm{RCo}_{4} \mathrm{~B}$ structure has two $\mathrm{R}$ sites ( $1 \mathrm{a}$ and $1 \mathrm{~b}$ ), two others for Co atoms (2c and 6i) and one for boron (2d). It has been shown that the $\mathrm{Co} 2 \mathrm{c}$ site, in $\mathrm{ThCo}_{4} \mathrm{~B}$ is the only site, which exhibits a significant magnetic moment [12], whereas both of the Th and Co 6i sites carry a negligible magnetic moment. On the contrary, in $\mathrm{YCo}_{4} \mathrm{~B}$ both $\mathrm{Co} 2 \mathrm{c}$ and Co 6i sites do carry a magnetic moment. We present in this paper a DFT-based study on the electronic band structure, spin-density maps, magnetic moment in Th$\mathrm{Co}_{4} \mathrm{~B}$ using different schemes and Brillouin-zone integration methods as implemented in the Wien2k package [13].

\section{Computational Methods}

The $\mathrm{CeCo}_{4} \mathrm{~B}$ structure can be obtained from the $\mathrm{CaCu}_{5}$ structure if two different crystal lattice sites were introduced for the $\mathrm{Ce}(1 \mathrm{a}$ and $1 \mathrm{~b})$ in addition to two other sites for $\mathrm{Co}(2 \mathrm{c}$ and $6 \mathrm{i})$ and one for $\mathrm{B}(2 \mathrm{~d})$, as shown in Figure 1. Kuzma et al. [14] showed that the B for Co substitution in $\mathrm{RCo}_{5}(\mathrm{R}$ is a rare earth or $\mathrm{Y}$ ) structure leads to a series of compounds of the $\mathrm{R}_{n+1} \mathrm{Co}_{3 \mathrm{n}+5} \mathrm{~B}_{2 \mathrm{n}}$ type. $\mathrm{ThCo}_{4} \mathrm{~B}$ differs significantly from the $\mathrm{RCO}_{4} \mathrm{~B}$ where $\mathrm{R}$ is a rare earth, having much higher $\mathrm{c}$ lattice parameter but with the lattice parameter a close observed for $\mathrm{PrCo}_{4} \mathrm{~B}$ and $\mathrm{NdCo}_{4} \mathrm{~B}$ [2]. $\mathrm{ThCo}_{5}$ crystallizes in the $\mathrm{CaCu}_{5}$ structure (space groupP $6 / \mathrm{mmm}$, No. 191). The unit cell contains one formula unit. The experimental lattice constants used in our calculations are $\mathrm{a}=4.75 \AA$ and $\mathrm{c}=3.93 \AA$ [16]. The $\mathrm{ThCo}_{4} \mathrm{~B}$ has the $\mathrm{CeCo}_{4} \mathrm{~B}$ type of structure (space groupP6/mmm, No. 191). The unit cell contains two formula units, with the room temperature lattice parameters obtained from X-ray diffraction are $\mathrm{a}=5.088 \AA$ and $\mathrm{c}=7.003 \AA[17]$.

The Wein $2 \mathrm{~K}$ electronic structure code uses the FullPotential Linearized Augmented Plane Wave (FPLAPW) [18] based on the Density Functional Theory (DFT) [19]. The Local Density Approximation (LDA) of Perdew and Wang [20] and the Generalized Gradient Approximation (GGA) of Perdew, Burke and Ernzerhof [21] were used for correlation and exchange potentials as implemented in the Wien2k code [13]. Both core and valence states are calculated self-consistently, the core states fully relativistically for the spherical part of the potential, and the valence 


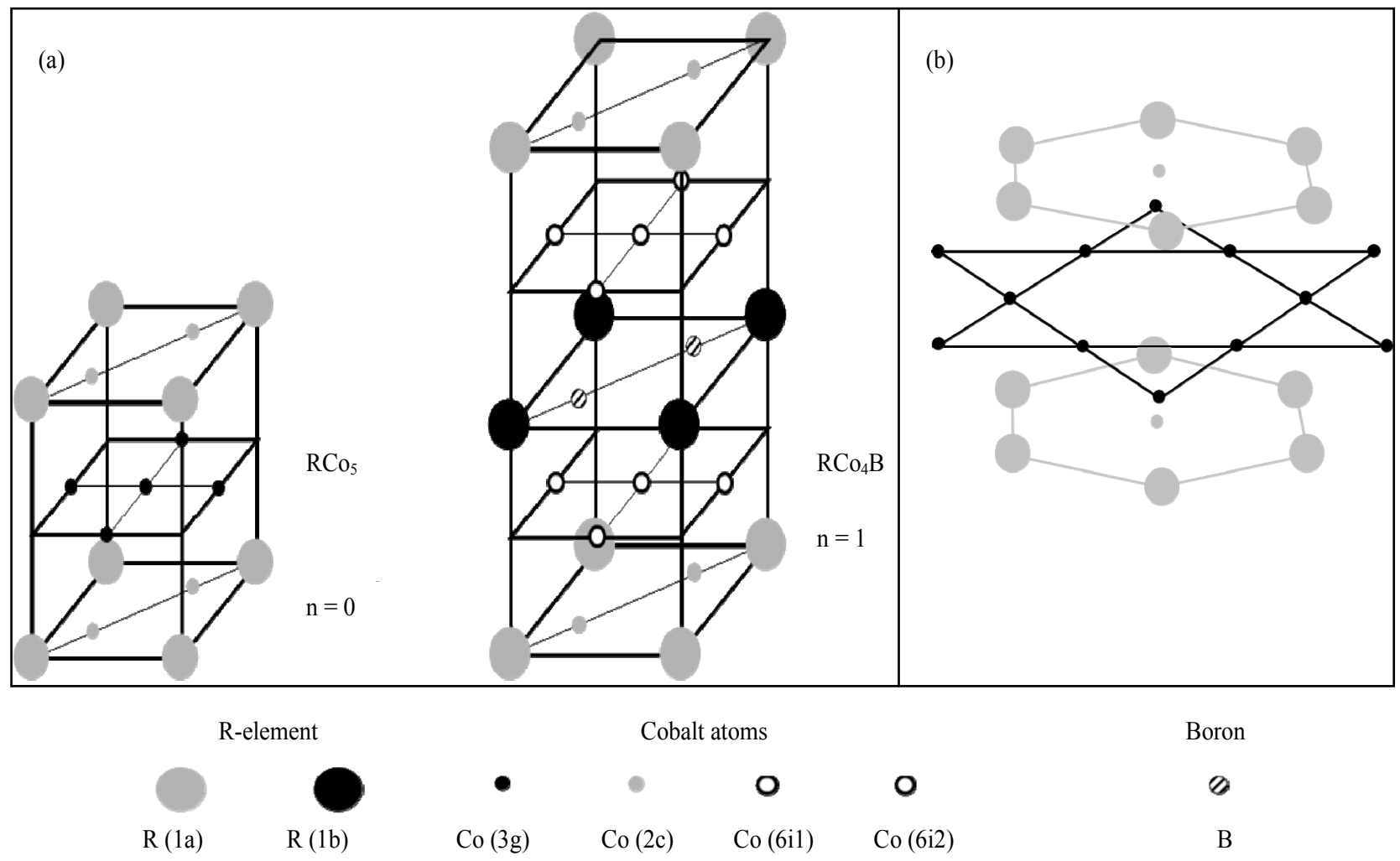

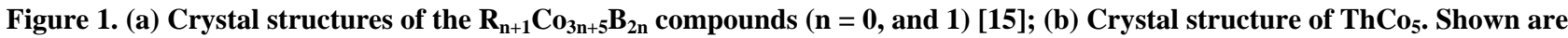
2 layers of hexagonal Co (2c) with a Kagome lattice of Co (3g) atoms lying between. The Th atoms lie in the middle of the hexagons in the Co (2c) layers [14].

states using the full potential. Local orbital extensions [22] with the converged basis of approximately 1000 basis functions were used to reduce linearization errors in Th and Co spheres. For the Brillouin zone integration we used the modified tetrahedron method for the self-consistent band structure calculations. Self-consistent calculations were performed and the convergence was checked by varying the number of $\mathrm{k}$ points up to 64 in the ireducible Brillouin zone. In order to systematically study the effect of considering different interactions on the magnetic properties, electronic bands and spin-density structure we first performed a pure spin-polarized calculation and then subsequently incorporated SO scheme into the calculation.

The SO interaction was included using a second variational scheme [23] by taking all states below the cut off energy 1.5 Ry. The orbital moment is affected by spin orbit coupling and other correlation effects. The band structure and density of states of localized electrons are not easy to be understood using local density approximation (LDA) method. The spin orbit interaction must be considered in dealing with the $6 \mathrm{p}$ states of Th. The $5 \mathrm{f}$ states however are above Fermi energy and, unlike the $4 \mathrm{f}$ states in rare-earth compounds, are not considered as localized.

\section{Results and Discussion}

The calculated magnetic moments at each sphere and their comparison with the experimental results and other calculation are shown in Table 1.

In this table we present our calculated magnetic moments at different $\mathrm{Th}$ and $\mathrm{Co}$ sites in $\mathrm{ThCo}_{5}$ and $\mathrm{ThCo}_{4} \mathrm{~B}$ structures together reported experimental and other $a b$ initio results for comparison. The calculated Co magnetic moments in $\mathrm{ThCo}_{4} \mathrm{~B}$ at the $2 \mathrm{c}$ and $6 \mathrm{i}$ sites of $\mathrm{Co}$ are 1.33 and $0.28 \mu_{\mathrm{b}}$ respectively. These values are in good agreement with 1.30 and $0.26 \mu_{\mathrm{b}}$ reported by Benea et al. [17] using the fully relativistic SPR-KKR method. Rather different values were reported by FPLO calculations [24] namely 1.548 and $0.427 \mu_{\mathrm{b}}$ respectively. Relatively different values were reported by neutron diffraction experiments [25] namely 1.8 and $0.1 \mu_{\mathrm{b}}$ respectively. It is also evident that the magnetic moments at the non-equivalent Th (1a) and Th (1b) sites and at the B (2d) site are rather small and negative in good agreement with earlier work $[17,24]$.The spin and site-resolved DOS for $\mathrm{ThCo}_{4} \mathrm{~B}$ and $\mathrm{ThCo}_{5}$ structures are shown in Figures 2(a) and (b) respectively. The main features of Figure 2 are:

a) The f-states of Th (1a) and Th (1b) non-equivalent sites contribute significantly to the total DOS of $\mathrm{ThCo}_{4} \mathrm{~B}$. These DOS states are located above $\mathrm{E}_{\mathrm{f}}$ indicating their 
Table 1. Magnetic moments in the ThCo $\mathrm{T}_{4} \mathrm{~B}$, and $\mathrm{ThCo}_{5}$ compounds.

\begin{tabular}{|c|c|c|c|c|c|c|c|}
\hline & \multirow{2}{*}{\multicolumn{2}{|c|}{$\begin{array}{c}\text { Co mag. mom. }(\mu \mathrm{B} / \text { atom }) \\
\text { Calc. }\end{array}$}} & \multirow{2}{*}{\multicolumn{2}{|c|}{$\begin{array}{c}\text { Co mag. mom. }(\mu \mathrm{B} / \text { atom }) \\
\text { Exp. }^{\mathrm{a}}\end{array}$}} & \multicolumn{3}{|c|}{ Magnetic moment ( $\mu \mathrm{B} /$ f.u. $)$} \\
\hline & & & & & \multirow[t]{2}{*}{ Calc. } & \multirow[t]{2}{*}{ Exp. $^{\text {a }}$} & \multirow[t]{2}{*}{ Exp. ${ }^{b}$} \\
\hline & Co $2 \mathrm{c}$ & Co $6 \mathrm{i}, 3 \mathrm{~g}$ & $\mathrm{Co} 2 \mathrm{c}$ & Co $6 \mathrm{i}, 3 \mathrm{~g}$ & & & \\
\hline \multirow{3}{*}{$\mathrm{ThCo}_{4} \mathrm{~B}$} & $1.30^{\mathrm{d}}[17]$ & $0.27^{\mathrm{d}}[17]$ & & & & & \\
\hline & $1.548^{\mathrm{e}}[24]$ & $0.427^{\mathrm{e}}[24]$ & $1.8[12]$ & $0.1[12]$ & $1.94^{\mathrm{d}}[17]$ & $2.1[12]$ & $1.5[12]$ \\
\hline & $1.332^{\mathrm{c}}$ & $0.281^{\mathrm{c}}$ & ---------- & ---------- & $2.137^{\mathrm{c}}$ & -------- & --------- \\
\hline $\mathrm{ThCo}_{5}(\mathrm{HMS})^{*}$ & $1.55^{\mathrm{d}}[17]$ & $1.48^{\mathrm{d}}[17]$ & $1.6[25]$ & $1.6[25]$ & $7.2^{\mathrm{d}}[17]$ & 8.0 & $7.3[26]$ \\
\hline $\mathrm{ThCo}_{5}(\mathrm{LMS})^{* *}$ & $1.10^{\mathrm{f}}[27]$ & $0.55^{\mathrm{f}}[27]$ & $1.2[25]$ & $1.0[25]$ & $4.2[27]$ & 5.4 & $4.9[26]$ \\
\hline \multirow[t]{3}{*}{$\mathrm{ThCo}_{5}$} & $1.201^{\mathrm{c}}$ & $1.353^{\mathrm{c}}$ & -------- & --------- & $5.857^{\mathrm{c}}$ & --------- & -------- \\
\hline & \multicolumn{7}{|c|}{ Magnetic moments ( $\mu \mathrm{B} /$ atom) for $\mathrm{ThCo}_{4} \mathrm{~B}$} \\
\hline & Th 1a & Th $1 b$ & Co $2 \mathrm{c}$ & Co $6 \mathrm{i}$ & B $2 d$ & & Total \\
\hline Ref.[17] & $-0.169^{\mathrm{d}}$ & $-0.167^{\mathrm{d}}$ & $1.106^{\mathrm{d}}$ & $0.252^{\mathrm{d}}$ & $-0.025^{\mathrm{d}}$ & & $1.670^{\mathrm{d}}$ \\
\hline Ref.[24] & $-0.161^{\mathrm{e}}$ & $-0.137^{\mathrm{e}}$ & $1.548^{\mathrm{e}}$ & $0.427^{\mathrm{e}}$ & $-0.043^{\mathrm{e}}$ & & ---------- \\
\hline This work & $-0.152^{\mathrm{c}}$ & $-0.139^{\mathrm{c}}$ & $1.332^{\mathrm{c}}$ & $0.281^{\mathrm{c}}$ & $-0.022^{\mathrm{c}}$ & & $1.644^{\mathrm{c}}$ \\
\hline
\end{tabular}

${ }^{\mathrm{a}}$ Neutron diffraction; ${ }^{\mathrm{b}}$ Magnetization measurements; ${ }^{\mathrm{c}}$ Current work; ${ }^{\mathrm{d}}$ Fully relativistic SPR-KKR calculations; ${ }^{\mathrm{e}}$ FPLO method; ${ }^{\mathrm{f}}$ Spin-orbit coupling included as perturbation. *High Moment Spin; ${ }^{* *}$ Low Moment Spin.

non-localized nature in these two compounds. The hybridization between the Th (1b) and B (2d) atoms results in DOS features different from those of the Th (1a) atoms. This may be attributed to the fact that Th (1a) atoms lack the presence of the B (2d) atoms in the crystal plane in which they are located;

b) The states of boron contribute but little to the total DOS. These features are generally in accord with the data reported by Benea et al. [17] and Szajek [24].

The calculated band structures of the two Co sites $2 \mathrm{c}$ and $6 \mathrm{i}$ have noticeable different behavior as shown in Figure 3. Considering the close relationship between the $\mathrm{ThCo}_{4} \mathrm{~B}$ and $\mathrm{ThCo}_{5}$ structure types (the ordered substitution of one half of the Co $2 \mathrm{c}$ site in the $\mathrm{ThCo}_{5}$ by B leads to $\mathrm{ThCo}_{4} \mathrm{~B}$ structure, with $\mathrm{B}$ located on the $2 \mathrm{~d}$ site), the calculated Co contributions to DOS can be compared in mentioned compounds. Upon changing the Co by B different environment were introduced for the Co atoms and its $3 \mathrm{~g}$ site in the $\mathrm{ThCo}_{5}$ structure to be the $6 \mathrm{i}$ site in the $\mathrm{ThCo}_{4} \mathrm{~B}$ structure. The effect of the SO coupling on the $6 p$ states of the Th (1a) and Th (1b) atoms is shown by comparing the spin-polarized Band Figure 4(a) to their counterparts where SO has been taken into consideration
Figure 4(b). The splitting into $6 \mathrm{p} 1 / 2$ and $6 \mathrm{p} 3 / 2$ is around $7 \mathrm{eV}$ however it is a bit larger for the Th (1a) atom. Our calculation is consistent with the work of Szajek [24].

The spin-density maps are shown in Figure 5, for $\mathrm{ThCo}_{4} \mathrm{~B}$ in the (100), (001) and (002) planes using just the spin-polarized scheme. In the (100) plane nine atoms of Th and four Co (2c) are displayed. The (100) and (001) planes are boron-free. The latter one displays 5 atoms of Th and 6 Co (2c) atoms. The (002) plane, on the other hand is Co-free and has $5 \mathrm{Th}$ and $6 \mathrm{~B}$ atoms (for the charge density maps see Figure 6). These spin-density maps show clearly that spin contours are concentrated mainly around the Co atoms in support to the data of Table 1. The effect of SO interaction on the spin density is shown in Figure 7. The SO effect is largely due to the p-state of the Th atoms as we have discussed earlier. We have performed similar calculation on $\mathrm{ThCo}_{5}$ in the (100) and (001) planes. These two planes contain $9 \mathrm{Th}+4 \mathrm{Co}$ and $5 \mathrm{Th}+6 \mathrm{Co}$ atoms respectively. We have found that the SO effect is also strongest on the Th atoms of this system. The spin-contour maps for $\mathrm{ThCo}_{5}$ in the (100) plane, using the bare spin-polarized approximation and in the case of incorporating the SO interaction are shown in Figures 8(a) and (b) respectively. 

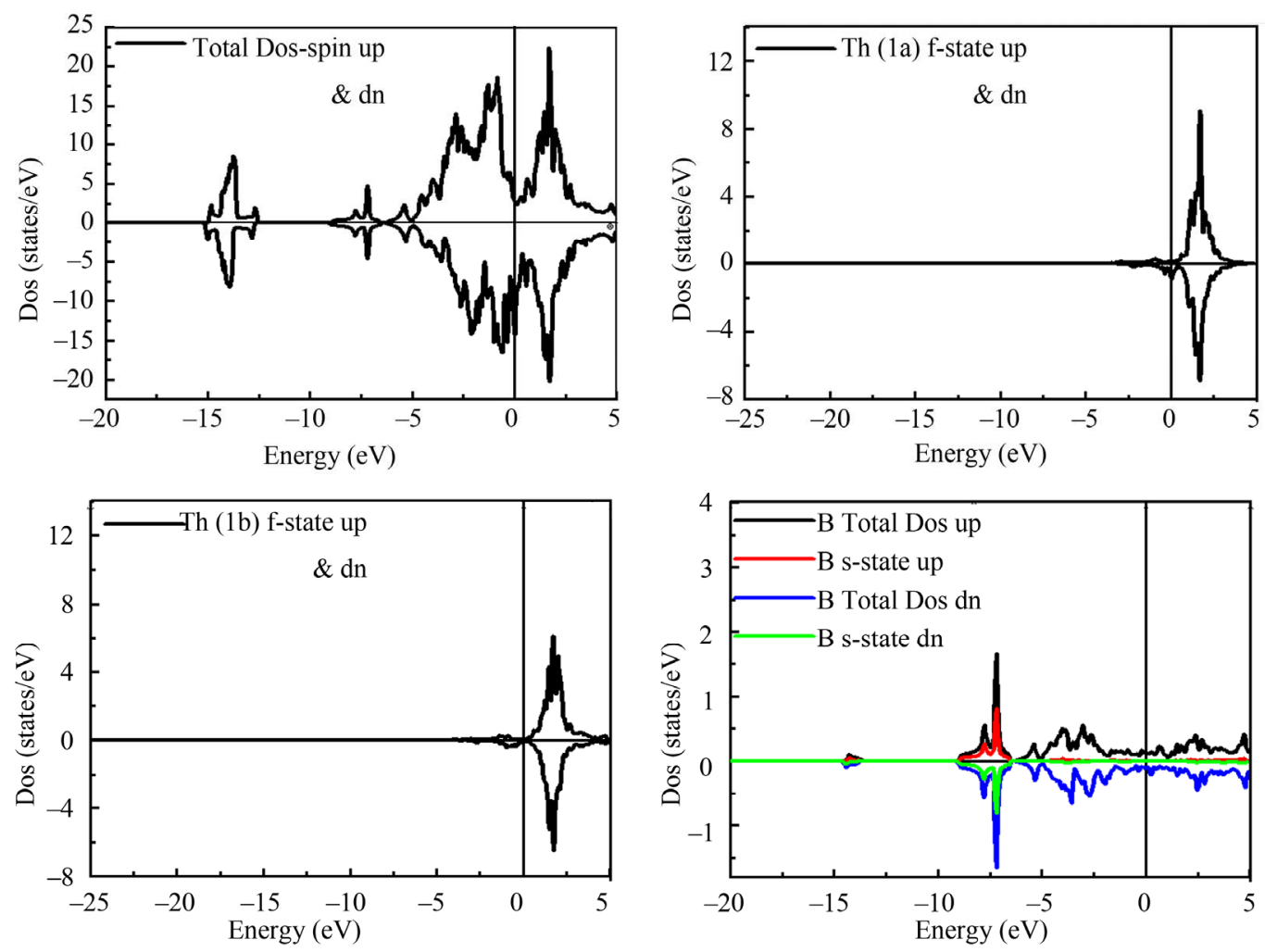

(a)
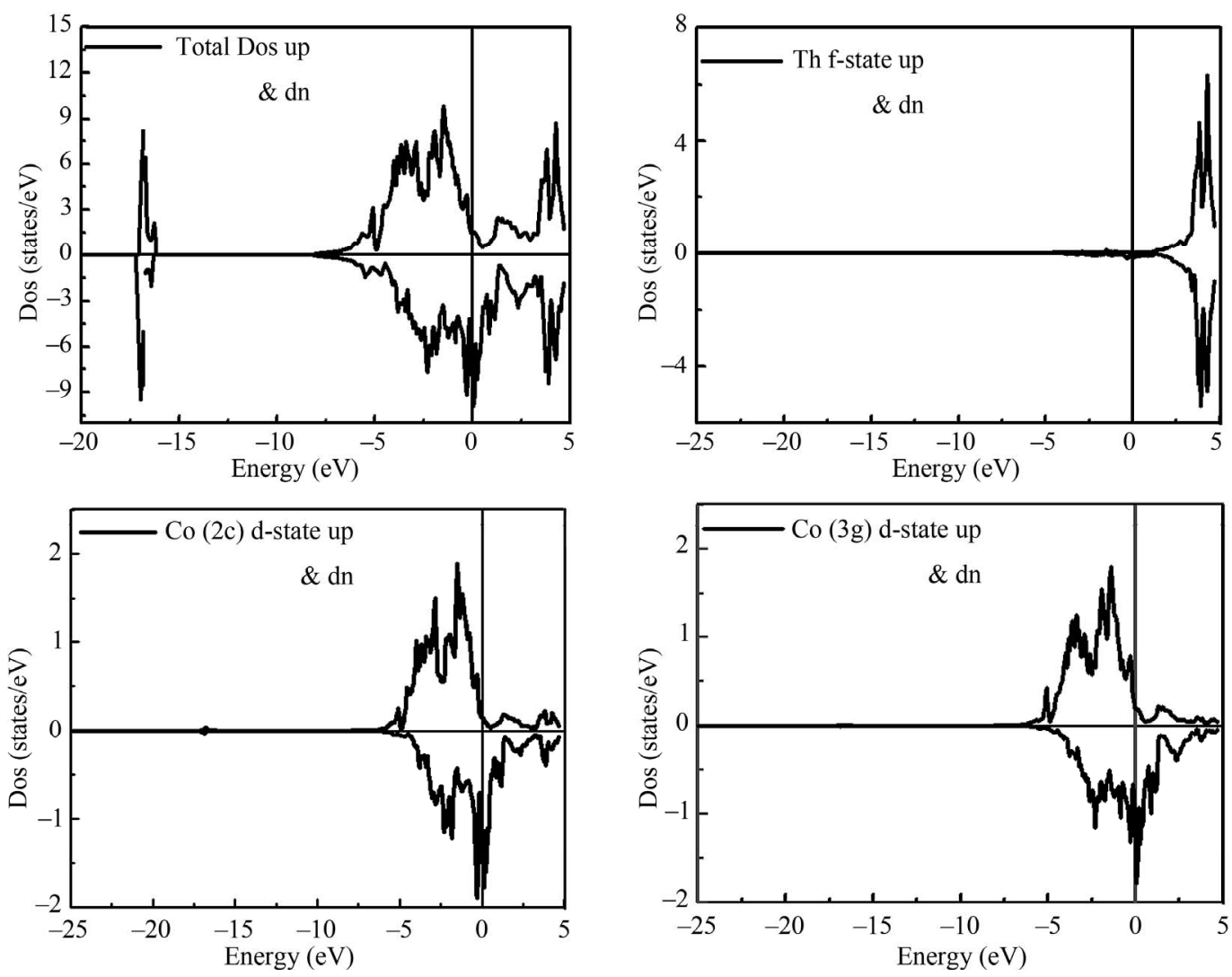

(b)

Figure 2. (a) the spin polarized Dos (up + down) for ThCo $\mathbf{0}_{4} B$; (b) the spin polarized Dos (up + down) for ThCo . 

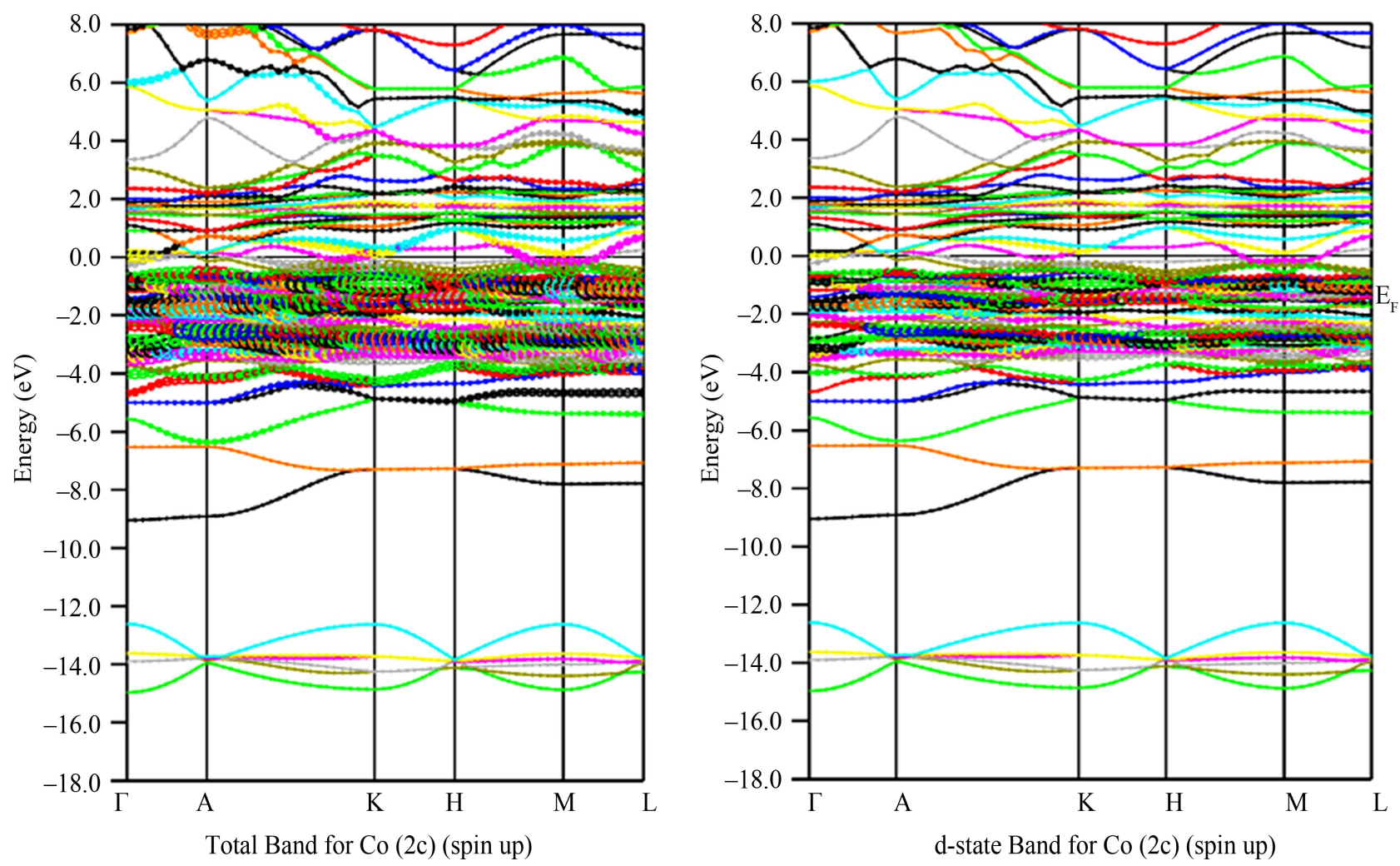

(a)
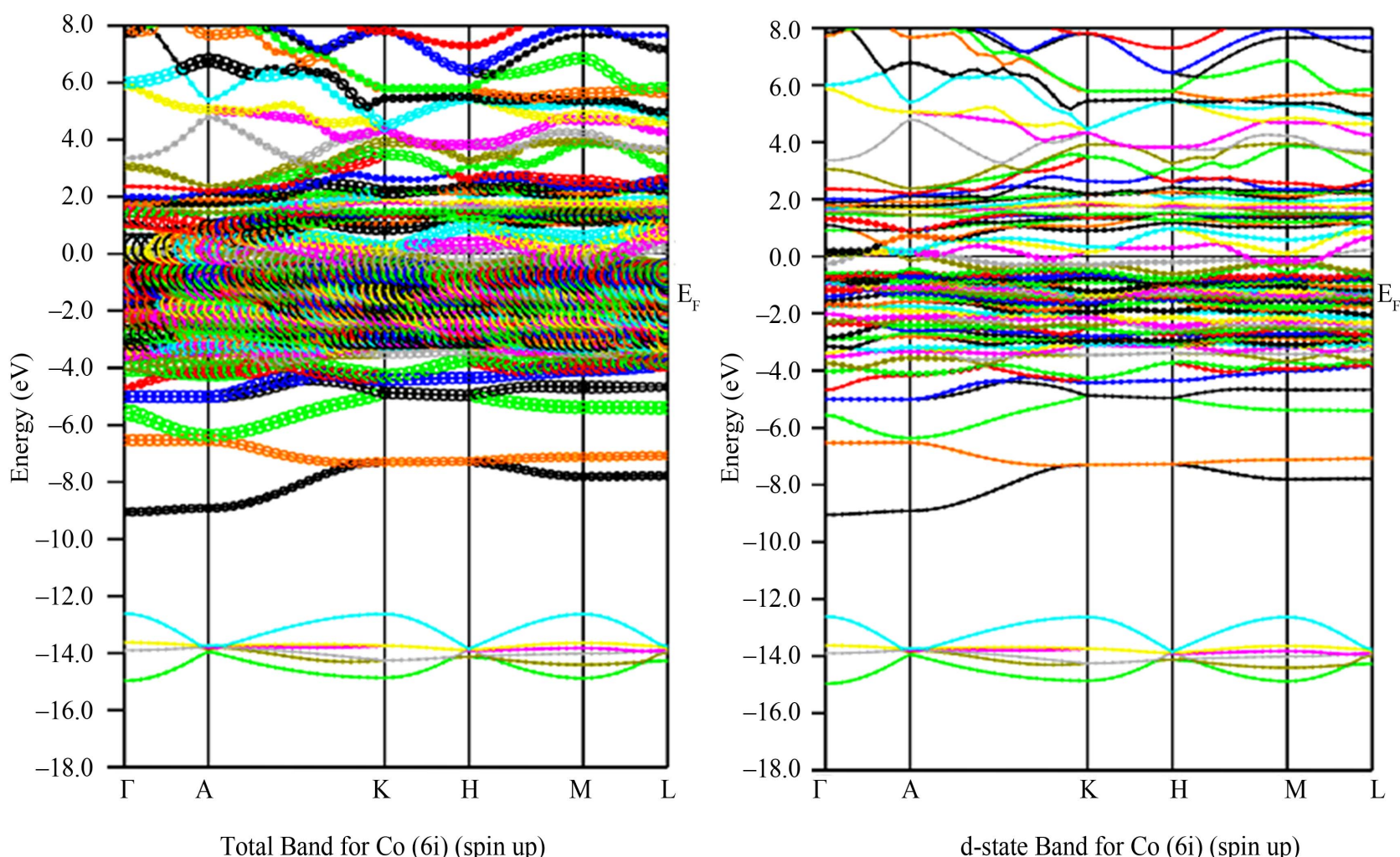

(b)

Figure 3. (a) the spin polarized band structure (up) for the Co (2c)-atom in $\mathrm{ThCo}_{4} \mathrm{~B}$; (b) the spin polarized band structure (up) for the $\mathrm{Co}(6 \mathbf{i})$-atom in $\mathrm{ThCo}_{4} \mathrm{~B}$. 

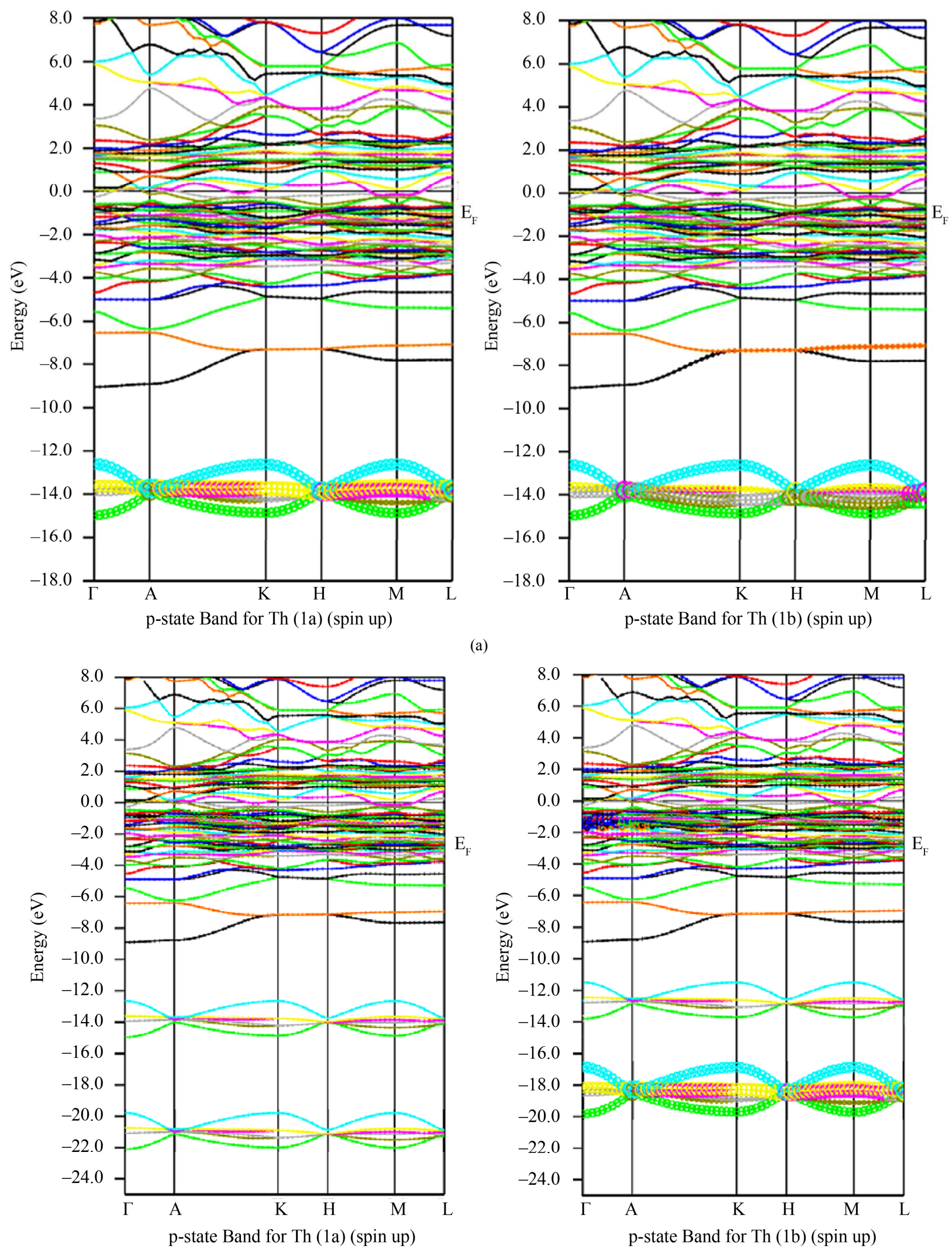

(a)

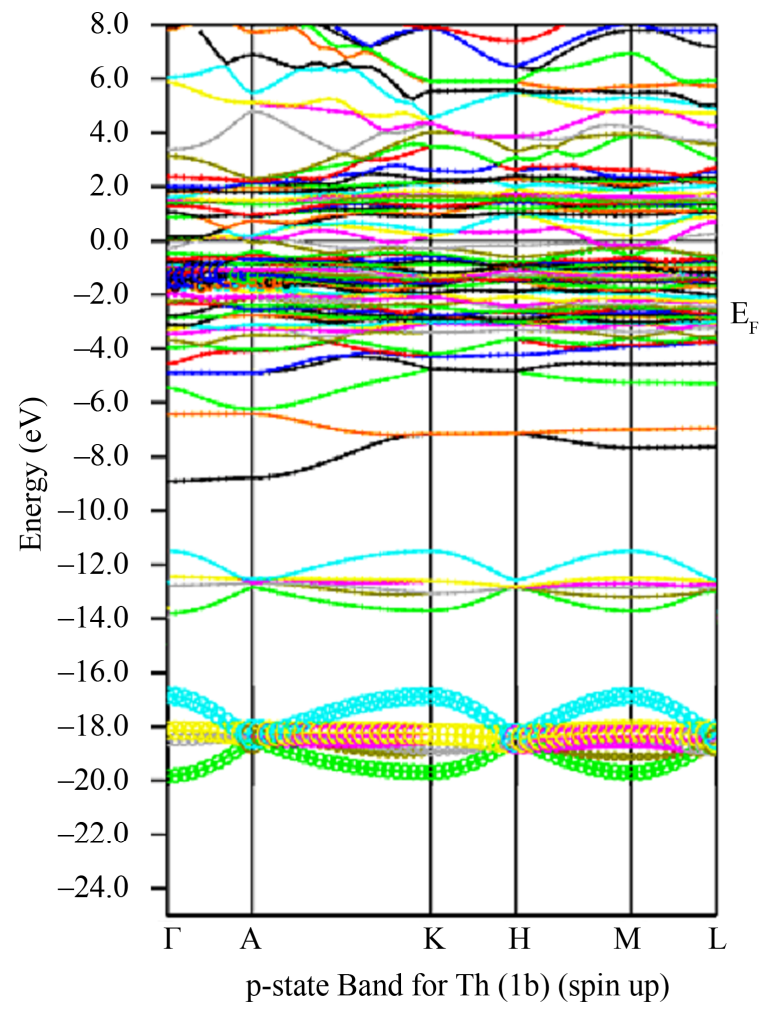

(b)

Figure 4. (a) the spin polarized band structure (up), for Th (1a) and Th (1b) [p-state] in ThCo $\mathrm{o}_{4} \mathrm{~B}$; (b) the spin polarized band structure with So (up) for Th (1a) \& Th (1b) [p-state] in ThCo $\mathrm{T}_{4} \mathrm{~B}$. 


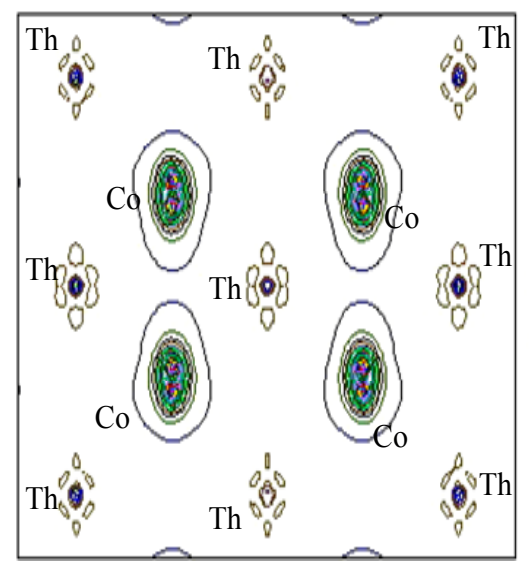

(a)

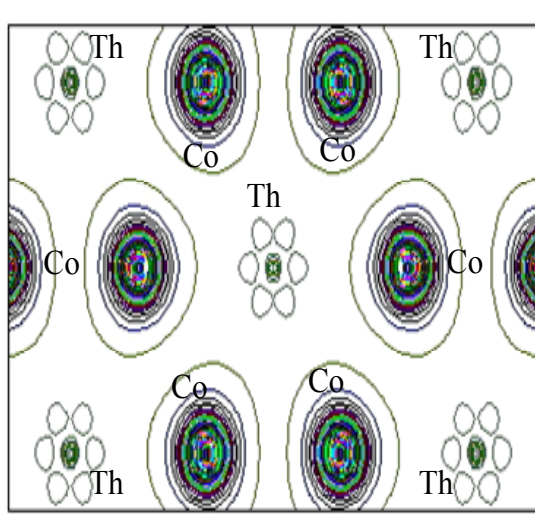

(b)

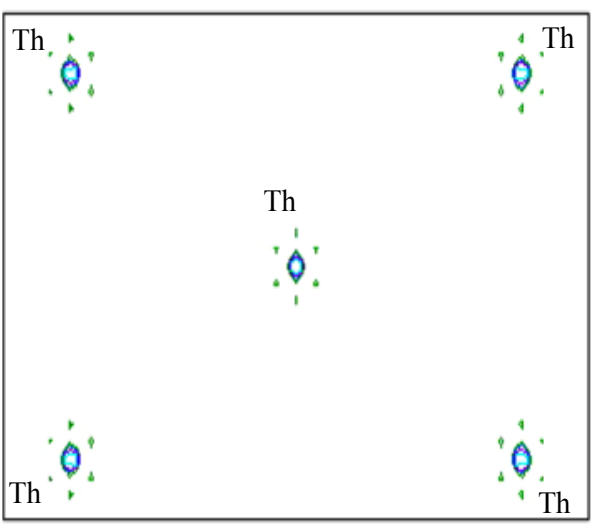

(c)

Figure 5. (a) the spin density map (spin polarized) for $\mathrm{ThCo}_{4} \mathrm{~B}$ (100-plane); (b) the spin density map (spin polarized) for $\mathrm{ThCo}_{4} \mathrm{~B}$ (001-plane); (c) the spin density map (spin polarized) for ThCo $\mathrm{o}_{4} \mathrm{~B}$ (002-plane).

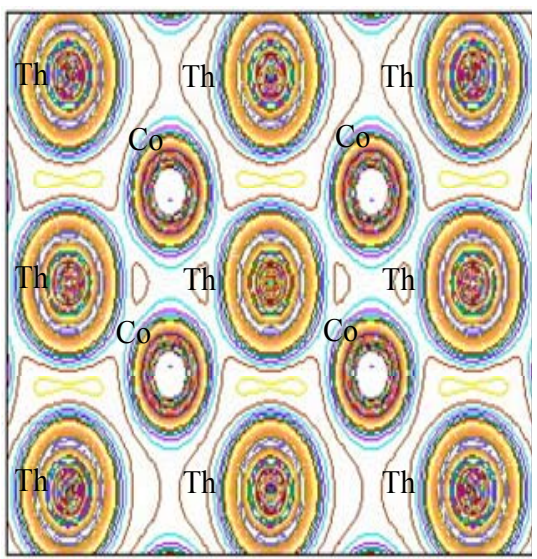

(a)

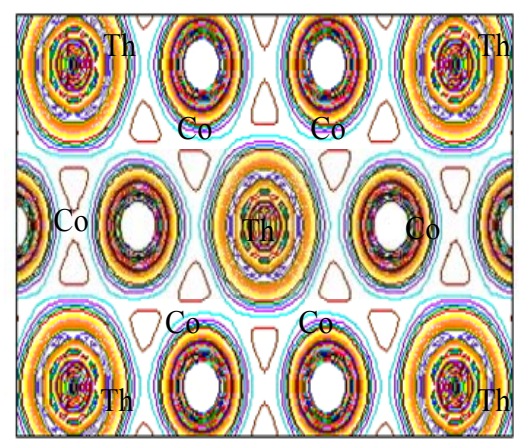

(b)

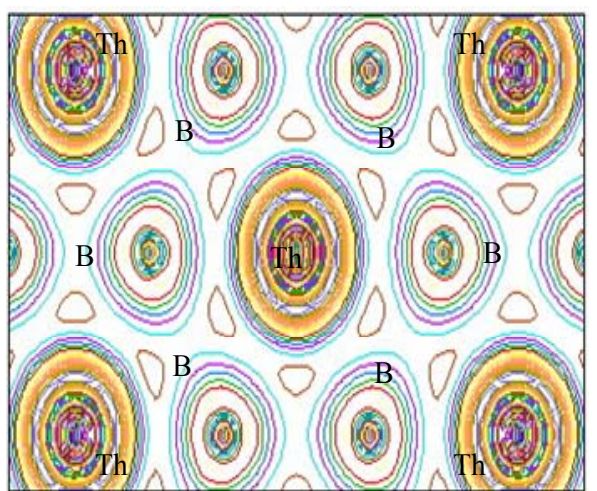

(c)

Figure 6. (a) the electronic charge density (spin polarized) for $\mathrm{ThCo}_{4} \mathrm{~B}$ (100-plane); (b) the electronic charge density (spin polarized) for $\mathrm{ThCo}_{4} \mathrm{~B}$ (001-plane); (c) the electronic charge density (spin polarized) for ThCo $\mathrm{o}_{4} \mathrm{~B}(002$-plane).

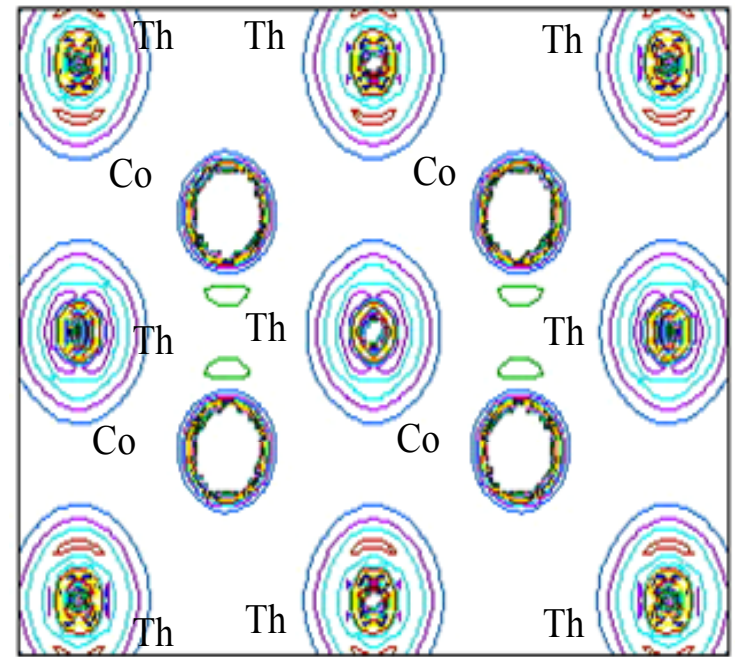

(a)

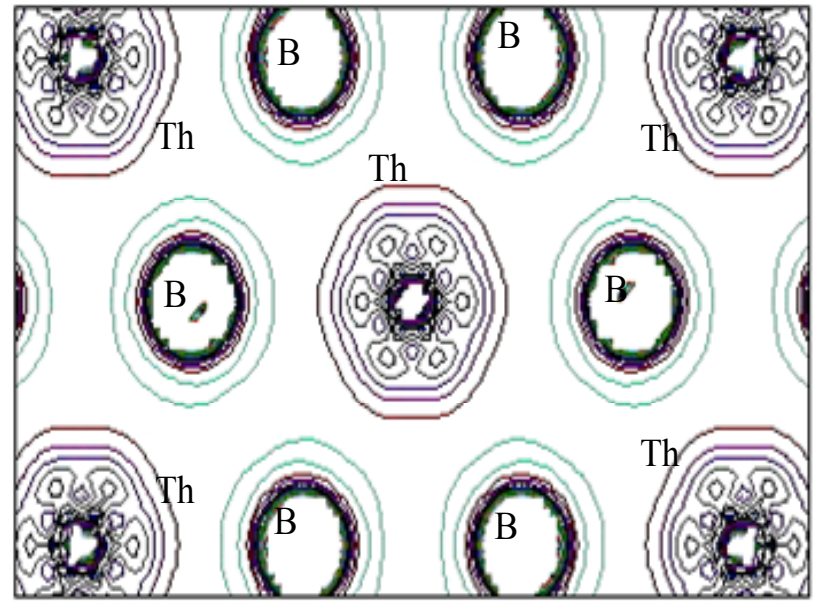

(b)

Figure 7. (a) the spin density map (spin polarized with SO) for $\mathrm{ThCo}_{4} \mathrm{~B}$ (100-plane); (b) the spin density map (spin polarized with SO) for $\mathrm{ThCo}_{4} \mathrm{~B}$ (002-plane). 

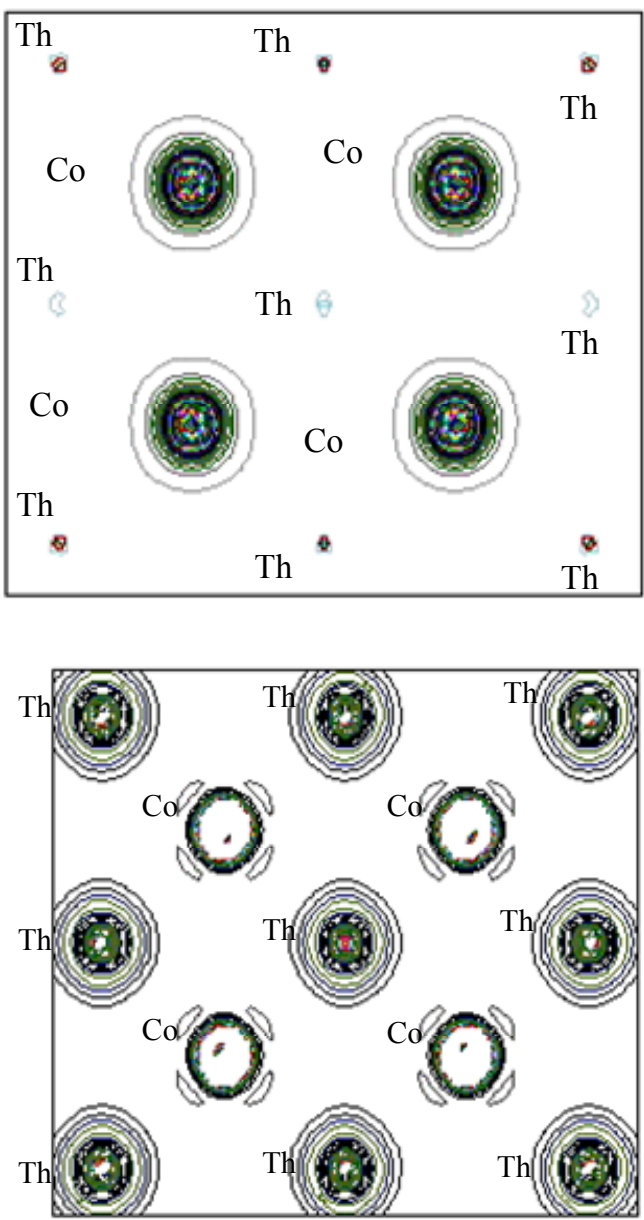

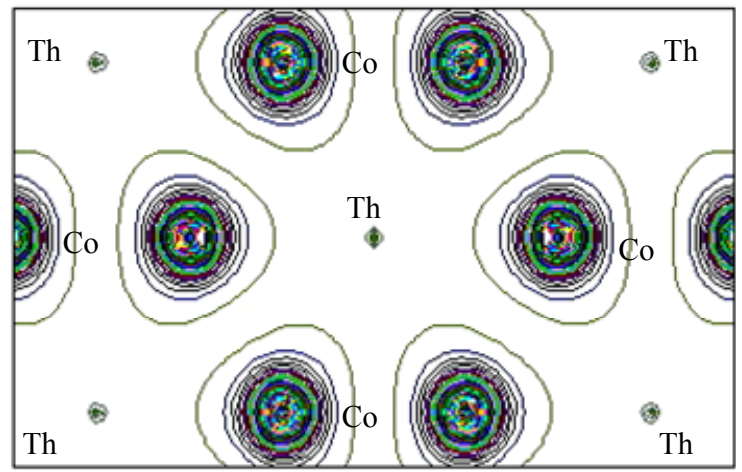

(a)

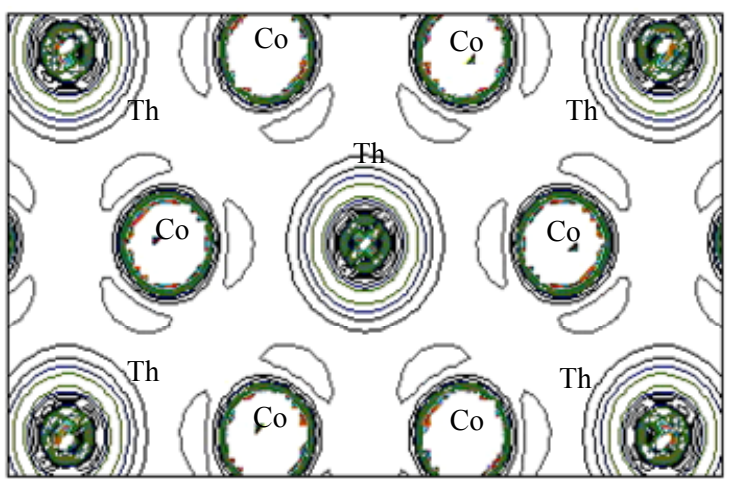

(b)

Figure 8. (a) (left) the spin density map (spin polarized) for $\mathrm{ThCo}_{5}(100$-plane). (right) the spin density map (spin polarized for) $\mathrm{ThCo}_{5}$ (001-plane); (b) (left) the spin density map (spin polarized with SO) for $\mathrm{ThCo}_{5}$ (100-plane). (right) the spin density

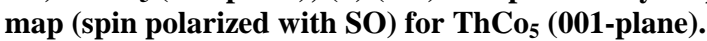

\section{Conclusion}

The magnetic moment of $\mathrm{ThCo}_{4} \mathrm{~B}$ compound is largely due to cobalt atoms in particular those at the $2 \mathrm{c}$ sites. Spin-orbit coupling affects the p-state of Th significantly. The $\mathrm{p}_{1 / 2}-\mathrm{p}_{3 / 2}$ spin-orbit splitting of this core state is site dependent, i.e. it is not the same for the Th (1a) and (1b) sites. Spin-polarized and spin-polarized calculation with SO interaction included, demonstrate the effect of SO on the spin density maps in $\mathrm{ThCo}_{4} \mathrm{~B}$ and its parent compound $\mathrm{ThCo}_{5}$.

\section{REFERENCES}

[1] C. V. Thang, L. H. Nam, N. P. Duong, et al., "Formation and Magnetic Properties of $\mathrm{RCo}_{4} \mathrm{Si}$ Compounds," Journal of Magnetism and Magnetic Materials, Vol. 196-197, 1999, pp. 765-767.

doi:10.1016/S0304-8853(98)00948-2

[2] C. Chacon and O. Isnard, "Magnetic Properties of the $\mathrm{RCo}_{4} \mathrm{~B}$ Compounds (R=Y, Pr, Nd, Sm, Gd, Tb)," Journal of Solid State Chemistry, Vol. 154, No. 1, 2000, pp. 242245. doi: $10.1006 /$ jssc. 2000.8843

[3] C. Zlotea and O. Isnard, "Neutron Diffraction and Magnetic Investigations of the $\mathrm{TbCo}_{4} \mathrm{M}$ Compounds $(\mathrm{M}=\mathrm{Al}$ and Ga)," Journal of Magnetism and Magnetic Materials, Vol. 253, No. 3, 2002, pp. 118-129. doi:10.1016/S0304-8853(02)00438-9

[4] C. Chacon and O. Isnard, "Magnetic Properties of the $\mathrm{RCo}_{4} \mathrm{~B}$ Compounds ( $\left.\mathrm{R}=\mathrm{Y}, \mathrm{Pr}, \mathrm{Nd}, \mathrm{Sm}, \mathrm{Gd}, \mathrm{Tb}\right)$," Journal of Solid State Chemistry, Vol. 154, No. 1, 2000, pp. 242-245. doi:10.1006/jssc.2000.8843

[5] P. Vlaic and E. Burzo, "Magnetic Properties and Electronic Structure of $\mathrm{Y}_{2} \mathrm{Co}_{7-\mathrm{x}} \mathrm{Fe}_{\mathrm{x}} \mathrm{B}$ Compounds," Moldavian Journal of the Physical Sciences, Vol. 1, 2002, p. 40.

[6] E. Burzo, V. Pop, C. Borodi and R. Ballou, "Magnetic Properties of $\mathrm{GdCo}_{4-\mathrm{x}} \mathrm{M}_{\mathrm{x}} \mathrm{B}$ Compounds where $\mathrm{M}=\mathrm{Fe}$ or Ni," IEEE Transactions on Magnetics, Vol. 30, No. 2, 1994, pp. 628-630. doi:10.1109/20.312357

[7] E. Burzo, V. Pop and N. Plugaru, "Magnetic Properties of $\mathrm{YCo}_{4-\mathrm{x}} \mathrm{Fe}_{\mathrm{x}} \mathrm{B}$ Compounds," Materials Science, Vol. 113, No. 2, 1989, pp. 253-256. 
[8] E. Burzo, V. Pop, N. Plugaru and I. Creanga, "Magnetic Properties of $\mathrm{YCo}_{4-\mathrm{x}} \mathrm{Fe}_{\mathrm{x}} \mathrm{B}$ Compounds," Physica Status Solidi (A), Vol. 113, 1989, p. 253.

[9] E. Burzo and M. Ursu, "Magnetic Properties of $\left(\mathrm{Gd}_{\mathrm{x}}\right.$ $\left.\mathrm{Y}_{1-\mathrm{x}}\right) \mathrm{Co}_{4} \mathrm{~B}$ Compounds," Journal of Magnetism and Magnetic Materials, Vol. 70, No. 1-3, 1987, pp. 345-346. doi:10.1016/0304-8853(87)90464-1

[10] A. Kowalczik, G. Chelkowska and A. Szajek, "X-Ray Photoemission Spectra and Electronic Structure of Gd$\mathrm{Co}_{4} \mathrm{~B}$," Solid State Communications, Vol. 120, No. 9-10, 2001, pp. 407-411. doi:10.1016/S0038-1098(01)00402-1

[11] A. Szajek and J. Morkowski, "Calculated Magnetic Moments and Electronic Structures of the Compounds $\mathrm{R}_{\mathrm{n}+1^{-}}$ $\mathrm{Co}_{3 \mathrm{n}+5} \mathrm{~B}_{2 \mathrm{n}}, \mathrm{R}=\mathrm{Gd}, \mathrm{Tb}, \mathrm{n}=0,1,2,3$, and $\infty$," Material Science Poland, Vol. 24, No. 3, 2006, p. 839.

[12] O. Isnard, V. Pop and J. C. Toussaint, "Neutron Diffraction Investigation of the Crystal and Magnetic Structure of the New $\mathrm{ThCo}_{4} \mathrm{~B}$ Compound," Journal of Physics: Condensed Matter, Vol. 15, No. 6, 2003, p. 791. doi:10.1088/0953-8984/15/6/306

[13] P. Blaha, K. Schwarz, G. K. H. Madsen, D. Kvasnicka, J. Luitz and Wien2k, "An Agumented Plane Wave + Local Orbitals for Calculating Crystal Properties," K. Schwarz, Techn. Universitat, Wien, 2001. http://www.wien2k.at/reg_user/index.html

[14] Y. B. Kuzma and N. Bilonizhko, "New Boride Structural Types in the Homologous Series Based on the $\mathrm{CaCu}_{5}$ and $\mathrm{CeCo}_{3} \mathrm{~B}_{2}$ Types," Soviet Physics - Crystallography, Vol. 18, 1974, pp. 447-449.

[15] O. Isnard, and C. Chacon Carillo, "Relation between Crystal Structure and Physical Properties of $\mathrm{R}_{\mathrm{n}+1} \mathrm{M}_{5+3 \mathrm{n}} \mathrm{B}_{2 \mathrm{n}}$ Phases," Journal of Alloys and Compounds, Vol. 442, No. 1-2, 2007, pp. 22-28. doi:10.1016/i.jallcom.2006.08.355

[16] J. Wang, Q. Guo and O. J. Kleppa, "Standard Enthalpies of Formation of Some Th Alloys with Group VIII Elements (Co, Ni, Ru, Rh, Pd, Ir and Pt), Determined by High-Temperature Direct Synthesis Calorimetry (Citations: 1)," Journal of Alloys and Compounds, Vol. 313, No. 1-2, 2000, pp. 77-84. doi:10.1016/S0925-8388(00)01157-9
[17] D. Benea, V. Pop and O. Isnard, "Electronic Structure and Magnetic Properties of the $\mathrm{ThCo}_{4} \mathrm{~B}$ Compound," Journal of Magnetism and Magnetic Materials, Vol. 320, No. 1-2, 2008, pp. 36-42. doi:10.1016/j.jmmm.2007.05.002

[18] D. J. Singh, "Planewaves, Pseudopotentials, and the LAPW Method," Kluwer Academic, Boston, 1994.

[19] W. Kohn and L. J. Sham, "Self-Consistent Equations Including Exchange and Correlation Effects," Physical Review A, Vol. 140, No. 4A, 1965, pp. 1133-1138.

[20] J. P. Perdew and Y. Wang, "Accurate and Simple Analytic Representation of the Electron-Gas Correlation Energy," Physical Review B, Vol. 45, No. 23, 1992, pp. 13244-13249. doi:10.1103/PhysRevB.45.13244

[21] J. P. Perdew, K. Burke and M. Ernzerhof, "Generalized Gradient Approximation Made Simple," Physical Review Letters, Vol. 77, No. 18, 1996, pp. 3865-3868. doi:10.1103/PhysRevLett.77.3865

[22] D. J. Singh, "Ground-State Properties of Lanthanum: Treatment of Extended-Core States," Physical Review B, Vol. 43, No. 6, 1991, pp. 6388-6392. doi:10.1103/PhysRevB.43.6388

[23] D. D. Koelling and B. Harmon, "Magnetic Properties of $\mathrm{SmCo}_{5}$ and $\mathrm{YCo}_{5}$," Journal of Physics C, Vol. 93, No. 10, 1977, p. 3107.

[24] A. Szajek, "Electronic and Magnetic Properties of Th$\mathrm{Co}_{4} \mathrm{~B}$," Acta Physica Polonica A, Vol. 113, No. 1, 2008, p. 283.

[25] D. Givord, J. Laforest and R. Lemaire, "Polarized Neutron Study of the Itinerant Electron Metamagnetism in ThCo $_{5}$," Journal of Applied Physics, Vol. 50, No. 11, 1979, pp. 7489-7491. doi:10.1063/1.326875

[26] D. Givord, J. Laforest, R. Lemaire and Q. Lu, "Cobalt Magnetism in $\mathrm{RCo}_{5}$-Intermetallics: Onset of 3d Magnetism and Magnetocrystalline Anisotropy ( $\mathrm{r}=$ Rare Earth or Th)," Journal of Magnetism and Magnetic Materials, Vol. 31, 1983, pp. 191-196.

[27] L. Nordstrom, B. Johansson, O. Eriksson and M. Brooks, "Theoretical Study of the Metamagnetism in $\mathrm{ThCo}_{5}$," Physical Review B, Vol. 42, No. 13, pp. 8367-8374. doi:10.1103/PhysRevB.42.8367 